\title{
Business Ethics - Wunscherfüller statt Heiler?
}

\author{
Im Rahmen der 7. Tagung der Deutschen Gesellschaft für Ästhetische Botulinumtoxin-Therapie e. V. \\ in Frankfurt sprach Dr. Keywan Taghetchian über Business Ethics in der ästhetischen Medizin und regt \\ damit zur Diskussion eines wichtigen Themas in der Ärzteschaft an.
}

? Worin unterscheidet sich das Verhältnis zwischen Arzt und Patient bei einer medizinisch indizierten und einer ästhetisch motivierten Behandlung?

Taghetchian: Hier gibt es einen wesentlichen Unterschied. In der ästhetischen Medizin gehen wir eher von Kunden aus, während in der klassischen Medizin von Patienten gesprochen wird. Patienten sind krank und stehen in einem Abhängigkeitsverhältnis zum Arzt. In diesem Abhängigkeitsverhältnis müssen sie vom Gesetzgeber auch stärker geschützt werden.

Ein Kunde ist nicht krank, er steht in keinem Abhängigkeitsverhältnis. Im Prinzip geht er aus freien Stücken zum Arzt. Allerdings kann er sich nicht alles wünschen, was er haben möchte, so wie es in der klassischen Dienstleistung der Fall ist. In der ästhetischen Medizin obliegt dem Arzt die Therapiefreiheit. Schlussendlich entscheidet er mit dem Kunden gemeinsam, was das Richtige für ihn ist. Somit sind Personen, die ästhetische Leistungen in Anspruch nehmen irgendwo zwischen Patienten und klassischen Kunden anzusiedeln.

? Welche Vorgaben und Gesetze sind bei der Betrachtung ethischer Aspekte des Arzt-Patienten-Verhältnisses im Bereich der ästhetischen Medizin von Bedeutung?

Taghetchian: Zum einen das Steuergesetz. Bei der Umsatzsteuer existieren Ausnahmeregelungen für Ärzte, die Patienten behandeln, denen ein Krankheitswert unterliegt. Diese Behandlungen sind von der Umsatzsteuer befreit. Behandlungen in der ästhetischen Medizin hingegen sind nicht von der Umsatzsteuer befreit. Hier postuliert der Gesetzgeber keinen zugrundeliegenden Krankheitswert.

Im Heilmittelwerbegesetz, in der Gebührenordnung für Ärzte und in der Medizinerberufsordnung verhält es sich hingegen anders. Personen, die Leistungen der ästhetischen Medizin in Anspruch nehmen, werden ebenso geschützt und unterliegen den gleichen Gesetzen wie Patienten der klassischen Medizin. Hier besteht eine gewisse Doppelmoral: Auf der einen Seite werden die Menschen, die zum ästhetischen Mediziner kommen, so behandelt, als ob sie keinen Krankheitswert haben und nicht geschützt werden müssen - auf der anderen Seite werden Sie behandelt, als ob sie einen Krankheitswert haben und vom Gesetz geschützt werden müssen.

? Wie könnte hier eine optimale Lösung aussehen?

Taghetchian: Ich bin auch der Meinung, dass ein ästhetischer Patient nicht krank ist, obwohl wir dem ästhetischen Menschen helfen, einen höheren Grad an Gesundheit zu erreichen. Ich beziehe mich dabei auf die Definition der Weltgesundheitsorgani-

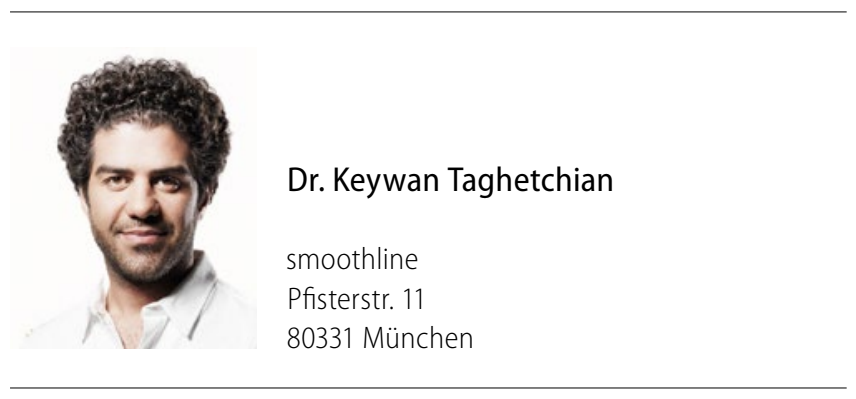

sation, die sagt: Gesundheit ist nicht nur das Fehlen von Krankheit und Gebrechen, sondern ein Zustand von vollständigem körperlichen und seelischem Wohlbefinden. Jene Menschen, die zu uns kommen und ästhetisch behandelt werden möchten, tun dies, weil sie sich besser fühlen möchten. Wir verhelfen diesen, einen höheren Grad an Gesundheit zu erlangen. Aber es besteht kein Krankheitswert. Daher stimme ich zu, nicht von der Umsatzsteuerpflicht befreit zu sein.

Wenn es um Regulierungen im Heilmittelwerbegesetz geht, das Arzneimittelgesetz und die medizinische Berufsordnung, müsste konsequenterweise auch hier kein Krankheitswert unterstellt werden. Der Mensch, der zum Ästhetiker geht, müsste also vom Gesetzgeber nicht so stark geschützt werden wie ein von der klassischen Medizin abhängiger Patient. Daher sollten in der ästhetischen Medizin gewisse Lockerungen stattfinden, damit ein ästhetischer Mediziner im Sinne eines ehrbaren Kaufmanns auch aktiver seine Kunst in der Gesellschaft bewerben darf.

? Existiert eine allgemeingültige Definition von „ästhetischer Dermatologie" und würde eine solche aus Ihrer Sicht Sinn ergeben?

Taghetchian: Die ästhetische Medizin ist nicht nur auf die Dermatologie beschränkt, daher halte ich eine solche Definition nicht für sinnvoll. Im Prinzip braucht es eine Neuerfindung dieses Gebiets, in dem minimalinvasiv ästhetisch behandelt wird, denn das ganze Gebiet der ästhetischen Medizin wird im Moment von allen Fachrichtungen mitbenutzt. Es bedarf einer eigenen Gesellschaft, die ein eigenes Berufsverständnis und eigene Wertvorstellungen von richtigem Handeln hat. Hier ist Pionierarbeit erforderlich, auch auf universitärer Ebene, beispielsweise mit einem Professorenstuhl.

Das Interview führte Ingo Schroeder. 